\title{
Ultrafast All Optical Switching via Tunable Fano Interference
}

\author{
Jin-Hui Wu, ${ }^{1,2, *}$ Jin-Yue Gao, ${ }^{1,2, \dagger}$ Ji-Hua Xu,${ }^{3}$ L. Silvestri, ${ }^{3}$ M. Artoni, ${ }^{4}$ G. C. La Rocca, ${ }^{3, \ddagger}$ and F. Bassani ${ }^{3}$ \\ ${ }^{1}$ College of Physics, Jilin University, Changchun 130023, People's Republic of China \\ ${ }^{2}$ Key Lab of CLAMS of Educational Ministry, Changchun 130023, People's Republic of China \\ ${ }^{3}$ Scuola Normale Superiore and INFM, Pisa, Italy \\ ${ }^{4}$ Department of Physics and Chemistry of Materials and LENS, Brescia University, Brescia, Italy
}

(Received 14 June 2004; published 25 July 2005)

\begin{abstract}
Tunneling induced quantum interference experienced by an incident probe in asymmetric double quantum wells can easily be modulated by means of an external control light beam. This phenomenon, which is here examined within the dressed-state picture, can be exploited to devise a novel all-optical ultrafast switch. For a suitably designed semiconductor heterostructure, the switch is found to exhibit frequency bandwidths of the order of $0.1 \mathrm{THz}$ and response and recovery times of about 1 ps.
\end{abstract}

DOI: 10.1103/PhysRevLett.95.057401

Transmission and switching of data using all-optical devices is currently the ultimate goal of most telecommunication research. To build all-optical networking systems, optical analogues of existing microelectronics devices must clearly be found. Interesting proposals toward the realization of fast switches, for instance, have already been brought forward whereas important steps toward their actual implementation are now being made. Schemes based on dipole-dipole interactions [1], nonlinear Bragg diffraction [2], photonic band-gap materials [3], as well as those most recent ones based on quantum interference in atomic media [4-7] all seem to be quite promising.

This Letter describes a proof-of-principle study demonstrating that tunable tunneling induced quantum interference in asymmetric quantum wells can be exploited to devise an efficient new mechanism for ultrafast and broadband all-optical switching working at low temperatures $(T \approx 10 \mathrm{~K})$. Unlike most familiar switching schemes based on photo-induced changes of the medium macroscopic refractive index [3] or other less usual ones based instead on virtual excitation of spin-polarized excitons [8] or on switchable mirrors made of thin polycrystalline films [9], this mechanism relies on quantum interference and hence it is quite sensitive. Quantum interference based phenomena such as, e.g., coherent population trapping [10], electromagnetically induced transparency [11], lasing without inversion [12], and light speed reduction [13] have recently attracted considerable attention. For many potential applications, solid-state solutions to implement these effects, first predicted and observed in dilute atomic media, are preferred and are now being sought after. In solid media [14] only few proposals followed the original idea of making use of quantum interference to devise an optical switch, first suggested [4] to work and recently observed [6] on a four-level atomic system.

We investigate the steady-state and transient behavior of a weak probe light signal incident upon an asymmetric quantum well. The probe can be stopped in the presence of an external control switch beam but made instead to propagate with little absorption when the switch beam is off. The
PACS numbers: 78.67.De, 42.50.Gy, 42.50.Hz, 78.66.Fd

different response depends, respectively, on whether tunneling induced quantum interference is quenched or well developed. Such a control of quantum interference hinges on the creation of a new dipole allowed intersubband transition. Large probe bandwidths $(\sim 0.1 \mathrm{THz})$ in the midinfrared range are obtained with $\mathrm{GaAs} / \mathrm{Al}_{x} \mathrm{Ga}_{1-x} \mathrm{As}$ [15] asymmetric quantum wells where appreciable on-off switching ratios are reached in just about one picosecond.

Our asymmetric double quantum well structure is shown in Fig. 1. The ground and first excited states that one would observe, respectively, in the shallow and deep wells when

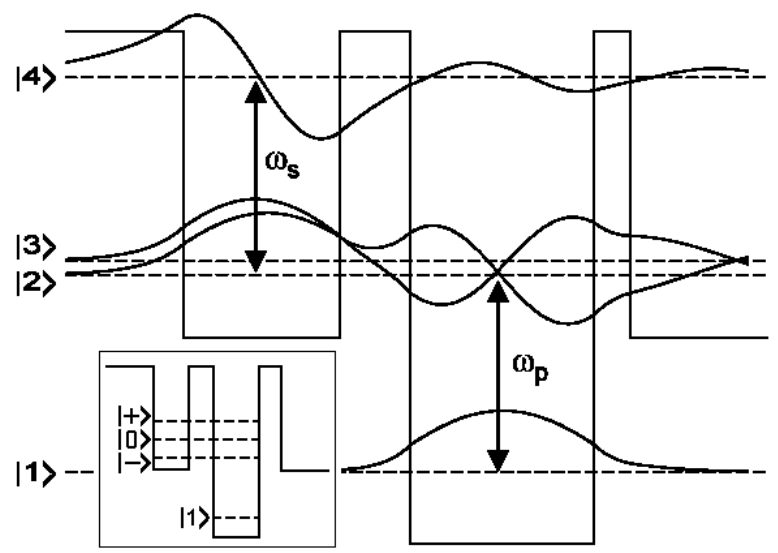

FIG. 1. Conduction subbands of the asymmetric double quantum well in the bare state picture. The deep well ground state $|1\rangle$ and the shallow well first excited state $|4\rangle$ have energies $E_{1}=$ $46.7 \mathrm{meV}$ and $E_{4}=296.3 \mathrm{meV}$, while the delocalized levels $|2\rangle$ and $|3\rangle$ have energies $E_{2}=174.8 \mathrm{meV}$ and $E_{3}=183.5 \mathrm{meV}$. The solid curves represent the corresponding wave functions. The growth sequence of the structure from left to right is as follows. A thick $\mathrm{Al}_{0.4} \mathrm{Ga}_{0.6} \mathrm{As}$ barrier is followed by a $\mathrm{Al}_{0.16} \mathrm{Ga}_{0.84} \mathrm{As}$ shallow well $(6.8 \mathrm{~nm})$. This shallow well is separated from the GaAs deep well $(7.7 \mathrm{~nm})$ on the right by a $\mathrm{Al}_{0.4} \mathrm{Ga}_{0.6} \mathrm{As}$ potential barrier $(3.0 \mathrm{~nm})$. Finally, a thin $(1.5 \mathrm{~nm})$ $\mathrm{Al}_{0.4} \mathrm{Ga}_{0.6} \mathrm{As}$ barrier separates the deep well from the last thick layer of $\mathrm{Al}_{0.16} \mathrm{Ga}_{0.84} \mathrm{As}$ on the right. Inset: in the presence of the switch field, subbands $|2\rangle$ and $|3\rangle$ couple with subband $|4\rangle$ giving rise to the three dressed states $|+\rangle,|0\rangle$, and $|-\rangle$. 
isolated mix to create two new states $|2\rangle$ and $|3\rangle$. Two external light fields of frequencies $\omega_{s}$ and $\omega_{p}$, playing the role of the switch and probe beam, couple in turn these two intermediate states, respectively, to the first excited state $|4\rangle$ of the shallow well and to the ground state $|1\rangle$ of the deep well. The latter state is the only one which is populated by electrons via $n$ doping by remote impurities. Tunneling to a continuum of energies takes place from states $|2\rangle$ and $|3\rangle$ through the thin barrier on the right. In the absence of the switch beam, the probability amplitude for the absorption of a probe photon can be thought of as the superposition of two absorption paths, one via level $|2\rangle$ and one via level $|3\rangle$, both decaying by tunneling to the same continuum. Fano-type destructive interference between the two absorption paths may then occur so as to cancel the probe absorption altogether. Nearly vanishing absorptions due to the Fano effect have already been predicted [16] and observed [17,18]. Yet in the presence of the switch field, the probe propagation properties are substantially modified by absorption. These modifications will be studied by adopting first a straightforward approach [16] that will then be compared with numerical modeling results $[19,20]$. The straightforward approach, which favors the physical insight into the nature of the probe propagation mechanism, is based on coupled Schrödinger-Maxwell equations

$$
\begin{aligned}
& \frac{\partial a_{1}}{\partial t}=j \Omega_{p}\left(a_{2}+q a_{3}\right) \\
& \frac{\partial a_{2}}{\partial t}=j \Omega_{p}^{*} a_{1}+j \Omega_{s} a_{4}+j\left[\left(\Delta_{p}+\Delta\right)+j \gamma_{2}\right] a_{2}+\gamma_{23} a_{3} \\
& \frac{\partial a_{3}}{\partial t}=j q \Omega_{p}^{*} a_{1}+j k \Omega_{s} a_{4}+j\left[\left(\Delta_{p}-\Delta\right)+j \gamma_{3}\right] a_{3}+\gamma_{23} a_{2} \\
& \frac{\partial a_{4}}{\partial t}=j \Omega_{s}^{*}\left(a_{2}+k a_{3}\right)+j\left[\left(\Delta_{p}+\Delta_{s}\right)+j \gamma_{4}\right] a_{4}
\end{aligned}
$$$$
\frac{\partial E_{p}(z, t)}{\partial z}+\frac{1}{c} \frac{\partial E_{p}(z, t)}{\partial t}=\frac{j \omega_{p}}{2 \varepsilon_{0} c} P_{p}(z, t)
$$

where $E_{p}(z, t)$ is the slowly varying electric field amplitude for a probe beam propagating in the " $z$ " direction and likewise for the polarization $P_{p}(z, t)$. As in the experiments in $[17,18]$, we consider a TM polarized probe incident at an angle of 45 degrees with respect to the growth axis so that all transition dipole moments include a factor $1 / \sqrt{2}$ as intersubband transitions are polarized along the growth axis. Here, $2 \Delta=\omega_{31}-\omega_{21}$ represents the frequency splitting between levels $|2\rangle$ and $|3\rangle$, while $\Delta_{p}=\omega_{p}-$ $\left(\omega_{21}+\omega_{31}\right) / 2$ and $\Delta_{s}=\omega_{s}-\left(\omega_{42}+\omega_{43}\right) / 2$ denote the probe and switch field detunings. The corresponding Rabi frequencies are instead given, respectively, as $\Omega_{p}=$ $E_{p} \mu_{12} / 2 \hbar$ and $\Omega_{s}=E_{s} \mu_{24} / 2 \hbar$, while $q=\mu_{13} / \mu_{12}$ and $k=\mu_{34} / \mu_{24}$ represent the ratio between the relevant subband transition dipole moments. Furthermore, $a_{i}(t)$ denotes the probability amplitude of finding the electron in level $|i\rangle$ whose decay rate $\gamma_{i}$ and the cross-coupling term $\gamma_{23}$ have been added phenomenologically.

In semiconductor quantum wells, the half linewidth $\gamma_{i}$ comprises a population-decay contribution $\gamma_{i l}$ as well as a dephasing contribution $\gamma_{i d}$ or $\gamma_{i}=\gamma_{i l}+\gamma_{i d}$. The latter may originate not only from electron-electron scattering and electron-phonon scattering, but also from inhomogeneous broadening due to scattering on interface roughness. For temperatures up to $10 \mathrm{~K}$ with electron sheet densities smaller than $10^{12} \mathrm{~cm}^{-2}$, the dephasing rates can be estimated [17] to be $\gamma_{2 d}=0.68 \mathrm{meV}, \gamma_{3 d}=0.80 \mathrm{meV}$, and $\gamma_{4 d}=0.50 \mathrm{meV}$. The population-decay rates can be calculated [21]: upon solving the effective mass Schrödinger equation with outgoing waves at infinity, we obtain a set of complex eigenvalues whose real and imaginary parts yield, respectively, the quasibound state energy levels and resonance widths. For our asymmetric double quantum well structure, the latter turn out to be $\gamma_{2 l}=3.47 \mathrm{meV}, \gamma_{3 l}=$ $4.13 \mathrm{meV}$, and $\gamma_{4 l}=0.80 \mathrm{meV}$. Finally, $\gamma_{23}=\sqrt{\gamma_{2 l} \gamma_{3 l}}$ that appears in Eqs. (1) describes the cross coupling between levels $|2\rangle$ and $|3\rangle$ arising from the tunneling to the continuum through the thin barrier next to the deep well. If $\varepsilon=\gamma_{23} / \sqrt{\gamma_{2} \gamma_{3}}$ is used to assess the strength of the cross coupling, where the limit values $\varepsilon=0$ and $\varepsilon=1$ correspond, respectively, to no interference and perfect interference, we obtain from the above estimates $\varepsilon=0.84$. This is close to the ideal value $\varepsilon=1$ and corresponds to a large tunneling efficiency leading to a strong Fano-type interference effect. As observed in Fig. 2(a) the efficiency is
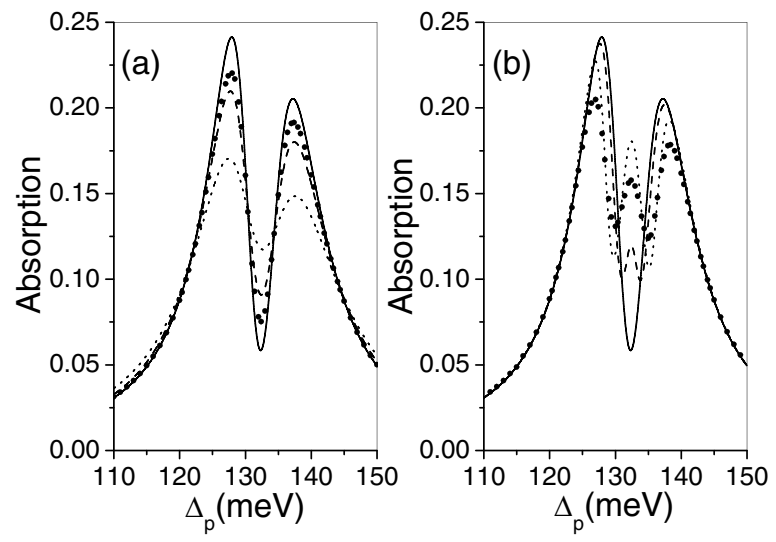

FIG. 2. (a) Probe absorption spectra from Eq. (3) with $\gamma_{2 d}=$ $0.68 \mathrm{meV}$ and $\gamma_{3 d}=0.80 \mathrm{meV}$ (solid line), with $\gamma_{2 d}=$ $1.36 \mathrm{meV}$ and $\gamma_{3 d}=1.60 \mathrm{meV}$ (dashed line), and with $\gamma_{2 d}=$ $2.72 \mathrm{meV}$ and $\gamma_{3 d}=3.20 \mathrm{meV}$ (dotted line). Other parameters are $\gamma_{2 l}=3.47 \mathrm{meV}, \gamma_{3 l}=4.13 \mathrm{meV}, \Omega_{s}=\Delta_{s}=0$, and $\Delta=$ $4.36 \mathrm{meV}$. The black dots represent computational results [20] (see text). (b) Probe absorption spectra from Eq. (3) with $\Omega_{s}=0$ (solid line), $\Omega_{s}=1.0 \mathrm{meV}$ (dashed line), and $\Omega_{s}=2.0 \mathrm{meV}$ (dotted line). Other parameters are $\gamma_{2 l}=3.47 \mathrm{meV}, \gamma_{3 l}=$ $4.13 \mathrm{meV}, \quad \gamma_{4 l}=0.80 \mathrm{meV}, \quad \gamma_{2 d}=0.68 \mathrm{meV}, \quad \gamma_{3 d}=$ $0.80 \mathrm{meV}, \gamma_{4 d}=0.50 \mathrm{meV}, \Delta_{s}=0$, and $\Delta=4.36 \mathrm{meV}$. The black dots represent computational results [20] for $\Omega_{s}=$ $2.0 \mathrm{meV}$ (see text). 
reduced by a temperature increase which generally leads to larger dephasings $\gamma_{i d}$.

In the limit of a weak probe signal, almost all electrons will remain in subband $|1\rangle$ and hence we may assume that $\left|a_{1}\right| \cong 1$. Under this assumption, we can solve Eqs. (1) for the steady-state values of the probability amplitudes, from which one arrives at the following probe susceptibility,

$$
\chi\left(\omega_{p}\right)=\frac{K\left[(k-q)^{2} \Omega_{s}^{2}-\left(q^{2} A+B\right) C-j 2 q \gamma_{23} C\right]}{C\left(A B+\gamma_{23}^{2}\right)-\Omega_{s}^{2}\left[\left(k^{2} A+B\right)+j 2 k \gamma_{23}\right]},
$$

with $\quad K=N\left|\mu_{21}\right|^{2} / 2 \hbar \varepsilon_{0}, \quad A=\left(\Delta_{p}+\Delta\right)+j \gamma_{2}, \quad B=$ $\left(\Delta_{p}-\Delta\right)+j \gamma_{3}$, and $C=\left(\Delta_{p}+\Delta_{s}\right)+j \gamma_{4}$, with $N$ being the electron volume density. An explicit analytical expression that illustrates the basic physics underlying the switching mechanism can be obtained as follows. Owing to the resonant tunneling between the ground level of the shallow well and the first excited level of the deep well, the wave functions of subbands $|2\rangle$ and $|3\rangle$ are symmetric and antisymmetric combinations of those associated with the isolated deep and shallow wells and we can take $q=$ $-k=-1$ in Eq. (3). Moreover, if subband levels $|2\rangle$ and $|3\rangle$ are assumed to have similar decay rates $\left(\gamma_{2} \cong \gamma_{3}\right)$, the $\mathrm{cw}$ probe single-pass transmission can be readily evaluated either when the switch beam is off $\left(\Omega_{s}=0\right)$ or when it is on $\left(\Omega_{s} \neq 0\right)$ for switch and probe fields both resonant $\left(\Delta_{s}=\Delta_{p}=0\right)$. In the first case, we obtain

$$
T(z)=\exp \left(\frac{-N \sigma_{2} z\left[\gamma_{2}\left(\gamma_{2}-\gamma_{23}\right)\right]}{\Delta^{2}+\gamma_{2}^{2}-\gamma_{23}^{2}}\right),
$$

where $\sigma_{2}=\omega_{p}\left|\mu_{21}\right|^{2} / \varepsilon_{0} c \hbar \gamma_{2}$. In the second case, we get instead

$$
T(z)=\exp \left(\frac{-N \sigma_{2} z \gamma_{2}\left[2 \Omega_{s}^{2}+\gamma_{4}\left(\gamma_{2}-\gamma_{23}\right)\right]}{\gamma_{4}\left(\Delta^{2}+\gamma_{2}^{2}-\gamma_{23}^{2}\right)+2 \Omega_{s}^{2}\left(\gamma_{2}+\gamma_{23}\right)}\right) .
$$

For appreciable values of the propagation constant $\mathrm{N \sigma}_{2} z$, small dephasings play a key role in making the switching mechanism efficient. When tunneling induced depopulation is the dominant decay mechanism $\left(\gamma_{23} \rightarrow \gamma_{2}\right)$ the "open" switch transmission is close to unity [see Eq. (4)], whereas for very small depopulation rates $\gamma_{i l}$ dephasings $\gamma_{i d}$ dominate $\left(\gamma_{23} \ll \gamma_{2}\right)$ and $T(z)$ is always small for values of $\gamma_{2}$ of the order of $\Delta$ (in the meV range). In the presence of the switch field, on the other hand, the exponent on the right-hand side of Eq. (5) becomes large enough to make the quantum well structure opaque to the probe via quenching of the tunneling induced interference. For small dephasings, the critical switch intensity $\left(I_{s}\right)_{\text {crit }}$ can be directly estimated from Eq. (5) as the value at which $T(z)$ decreases to $1 / e$,

$$
\begin{aligned}
\left(I_{s}\right)_{\text {crit }} & =2 \varepsilon_{0} c\left(\frac{\hbar}{\mu_{24}}\right)^{2}\left(\Omega_{s}^{2}\right)_{\text {crit }} \\
& =2 \varepsilon_{0} c\left(\frac{\hbar}{\mu_{24}}\right)^{2} \frac{\gamma_{4} \Delta^{2} / 2 \gamma_{2}}{N \sigma_{2} z-2} .
\end{aligned}
$$

Typically, $\sigma_{2} \leq 10^{-13} \mathrm{~cm}^{2}$ and $N z \leq 10^{12} \mathrm{~cm}^{-2}$, and hence for a single period structure one has $N \sigma_{2} z \cong$ $10^{-2}$. In order to make $T(z)$ to decrease to $1 / e$ one should have $N \sigma_{2} z \geq 2$, a condition which can be achieved either by preparing samples with a large number of periods or by using a sample with several periods inside an optical resonator in a multiple-pass configuration. Critical intensities of the order of $10^{6} \mathrm{~W} / \mathrm{cm}^{2}$ require values of $N \sigma_{2} z \cong$ 5 while lower switching intensities $\left(I_{s}\right)_{\text {crit }}$ can be attained for smaller values of $\gamma_{4}$ and $\Delta$.

We proceed to examine probe absorption profiles for realistic semiconductor parameters. We report in Fig. 2 profiles obtained directly with the help of the susceptibility in Eq. (3) for different values of the dephasing rates and compare them with the ones obtained from numerical modeling [20], where the two-dimensional subband dispersions, temperature dependent electronic distribution functions, and electron-electron and electron-phonon scattering rates have been included. The comparison largely confirms the features of the spectra obtained from Eq. (3) which in turn justifies the alternative phenomenological approach adopted earlier. We can see from Fig. 2(a) that for small dephasings $\left(\gamma_{i d} \ll \gamma_{i l}\right)$ a resonant probe can propagate with little absorption (solid curve) when the switch beam is off. Yet when the dephasings $\gamma_{i d}$ become important, effects of tunneling induced interference start to become less pronounced, which results into a resonant probe being more and more absorbed (dashed and dotted curves). When the switch beam is on we observe instead from Fig. 2(b) that a third probe absorption peak appears whose height depends on the applied switching field strength. This can be readily explained in terms of dressed states. Taking $\left|2^{\prime}\right\rangle\left(\left|3^{\prime}\right\rangle\right)$ as the first excited (ground) state of the deep (shallow) well when they are isolated by an infinitely thick barrier one has, in the case of resonant tunneling $\left(\omega_{2}^{\prime}=\omega_{3}^{\prime}\right),|2\rangle=\left(\left|3^{\prime}\right\rangle+\left|2^{\prime}\right\rangle\right) / \sqrt{2}$ and $|3\rangle=$ $\left(\left|3^{\prime}\right\rangle-\left|2^{\prime}\right\rangle\right) / \sqrt{2}$. Because $\left\langle 2^{\prime}|\hat{\mu}| 1\right\rangle$ is the only nonvanishing probe dipole matrix element, it follows that subband levels $|2\rangle$ and $|3\rangle$ are both coupled by the probe to the ground with $\mu_{21} \equiv\langle 2|\hat{\mu}| 1\rangle=-\mu_{31}=\mu_{2^{\prime} 1} / \sqrt{2}$. Likewise for the switch one has $\mu_{42}=\mu_{43}=\mu_{43^{\prime}} / \sqrt{2}$. When $\Delta_{s}=0$ the switch couples subbands $|2\rangle$ and $|3\rangle$ with subband $|4\rangle$ and gives rise to three dressed states $|+\rangle,|0\rangle$, and $|-\rangle$ as shown in the inset of Fig. 1. The central one, in particular,

$$
|0\rangle=\frac{\cos \phi}{\sqrt{2}}(|2\rangle-|3\rangle)-\sin \phi|4\rangle \quad\left(\tan \phi=\frac{\Delta}{\sqrt{2} \Omega_{s}}\right)
$$

with eigenfrequency $\omega_{0}=\left(\omega_{2}+\omega_{3}\right) / 2$ may couple to the ground $|1\rangle$ causing the absorption of a resonant probe $\left(\Delta_{p}=0\right)$. This new dipole allowed transition $|0\rangle \leftrightarrow|1\rangle$ induced by the switch field has a dipole moment $\mu_{01}=$ $\cos \phi\left(\mu_{21}-\mu_{31}\right) / \sqrt{2}=\cos \phi \mu_{2^{\prime} 1}$ which depends on $\Delta$ and $\Omega_{s}$. As a consequence, the third absorption peak in Fig. 2(b) increases with increasing $\Omega_{s}$.

Figure 3 further shows the dependence of the probe transmission on the propagation constant $N \sigma_{2} z$ for various 

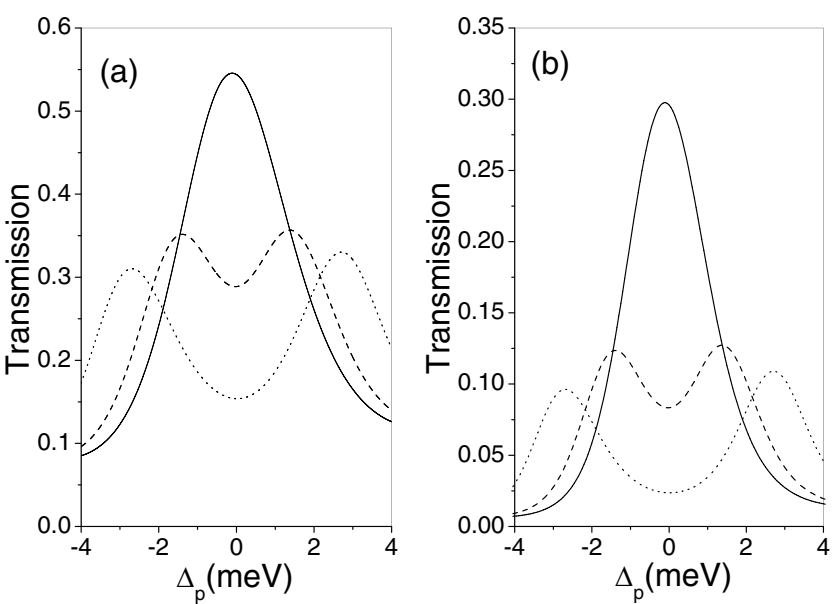

FIG. 3. Transmission spectra of the probe field for (a) $N \sigma_{2} z=5$ and (b) $N \sigma_{2} z=10$. Here $\Omega_{s}=0$ (solid line), $\Omega_{s}=1.0 \mathrm{meV}$ (dashed line), and $\Omega_{s}=2.0 \mathrm{meV}$ (dotted line) while other parameters are the same as in Fig. 2(b).

switch intensities. If $T_{o}$ and $T_{c}$ denote the probe transmission associated, respectively, with the open $\left(\Omega_{s}=0\right)$ or closed $\left(\Omega_{s} \neq 0\right)$ switch, then the ratio $\alpha=\left(T_{o}-\right.$ $\left.T_{c}\right) /\left(T_{o}+T_{c}\right)$ may be used as a measure of the on/off ratio. For $\Omega_{s}=2.0 \mathrm{meV}\left(\Omega_{s}=1.0 \mathrm{meV}\right)$, we obtain $\alpha=$ $0.56(\alpha=0.31)$ when $N \sigma_{2} z=5$ or $\alpha=0.85(\alpha=0.56)$ when one takes instead $N \sigma_{2} z=10$. A probe frequency bandwidth as large as $0.1 \mathrm{THz}$ associated with a significant

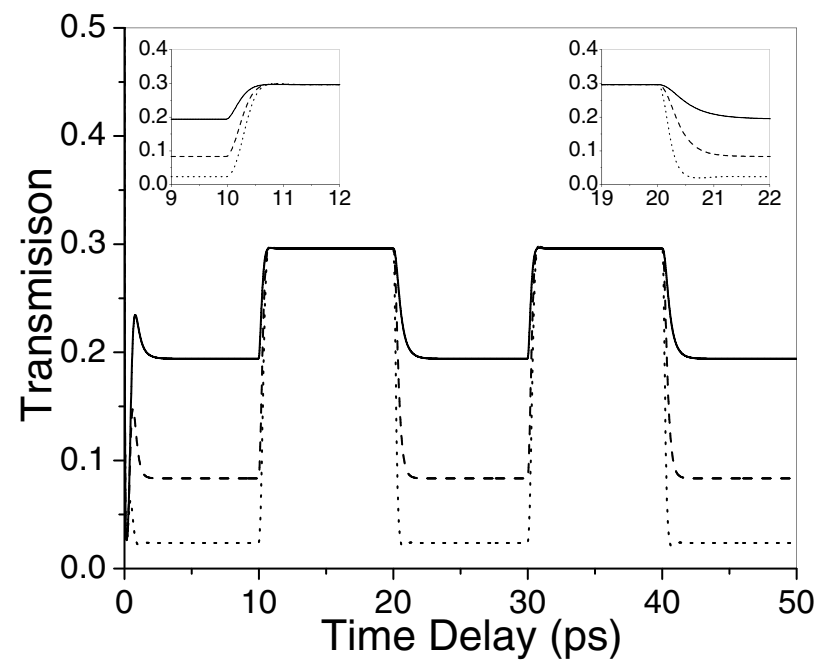

FIG. 4. Time evolution of a cw probe field with $\Delta_{p}=0, \Omega_{p}=$ $0.01 \mathrm{meV}$, and $N \sigma_{2} z=10$. Here $\Omega_{s}=0.5 \mathrm{meV}$ (solid line), $\Omega_{s}=1.0 \mathrm{meV}$ (dashed line), and $\Omega_{s}=2.0 \mathrm{meV}$ (dotted line) while other parameters are the same as in Fig. 2(b). The cw switching field is turned on or off at a period of 10 ps. Inset: transient blowup showing switching and recovery times of about 1 ps. differential transmittivity $\Delta T / T=\left(T_{o}-T_{c}\right) / T_{o} \simeq 0.73$ can be directly inferred from Fig. 3.

By numerically solving Eqs. (1) and (2), we can finally examine the time evolution of the probe transmission. The evolution is reported in Fig. 4 for a probe assumed to be a continuous wave and the switch beam a sharp square wave pulse train. Apart from a short initial transient, the transmitted probe either in the "open" or "close" position is seen to exhibit much the same stepwiselike profile reaching in both cases the steady-state value typically in less than one picosecond (see the inset of Fig. 4). This originates mainly from the fact that the population remains essentially in the ground level $|1\rangle$ at all times so that the control switch beam couples almost empty levels. Our proposal thus entails no real excitations and hence confirms the advantage of using quantum interference based schemes in ultrafast switching applications.

This work is supported by NSFC (Grants No. 10334010 and No. 10404009), MAE (Grant ST China-Italy), and MIUR (Accion Integrada IT-1603).

*Corresponding author.

Email: wujinhui0431@ sina.com

${ }^{\dagger}$ Email: jygao@mail.jlu.edu.cn

Email: larocca@sns.it

[1] A. S. Manka et al., Phys. Rev. Lett. 73, 1789 (1994).

[2] G. Pan, R. Kesavamoorthy, and S. A. Asher, Phys. Rev. Lett. 78, 3860 (1997).

[3] S. W. Leonard et al., Phys. Rev. B 66, 161102(R) (2002).

[4] S. E. Harris and Y. Yamamoto, Phys. Rev. Lett. 81, 3611 (1998).

[5] H. Schmidt and R. J. Ram, Appl. Phys. Lett. 76, 3173 (2000).

[6] M. Yan, E. G. Rickey, and Y. Zhu, Phys. Rev. A 64, 041801(R) (2001).

[7] C. Y. Ye et al., Opt. Lett. 28, 2133 (2003).

[8] E. Gansen et al., Appl. Phys. Lett. 80, 971 (2002).

[9] J.W. J. Kerssemakers et al., Nature (London) 406, 489 (2000).

[10] E. Arimondo, Progress in Optics $X X X V$, edited by E. Wolf (Elsevier Science, Amsterdam, 1996), p. 257.

[11] S.E. Harris, Phys. Today 50, No. 7, 36 (1997), and references therein.

[12] S. E. Harris, Phys. Rev. Lett. 62, 1033 (1989).

[13] L. V. Hau et al., Nature (London) 397, 594 (1999).

[14] X. M. Su and J. Y. Gao, Phys. Lett. A 264, 346 (2000).

[15] Other heterostructures such as, e.g., GaN $/ \mathrm{Al}_{x} \mathrm{Ga}_{1-x} \mathrm{~N}$ and $\mathrm{In}_{x} \mathrm{Ga}_{1-x} \mathrm{As} / \mathrm{AlAs}$, may likewise be used to make the switch work at different wavelengths.

[16] H. Schmidt et al., Opt. Commun. 131, 333 (1996).

[17] H. Schmidt et al., Appl. Phys. Lett. 70, 3455 (1997).

[18] J. Faist et al., Nature (London) 390, 589 (1997).

[19] M. E. Donovan et al., Phys. Rev. Lett. 87, 237402 (2001).

[20] L. Silvestri et al., Eur. Phys. J. B 27, 89 (2002).

[21] D. Ahn and S. L. Chuang, Phys. Rev. B 34, R9034 (1986). 